\title{
ON SUPERLINEAR $p(x)$-LAPLACIAN-LIKE PROBLEM WITHOUT AMBROSETTI AND RABINOWITZ CONDITION
}

\author{
Ge Bin
}

\begin{abstract}
This paper deals with the superlinear elliptic problem without Ambrosetti and Rabinowitz type growth condition of the form:

$\left\{\begin{array}{l}-\operatorname{div}\left(\left(1+\frac{|\nabla u|^{p(x)}}{\sqrt{1+|\nabla u|^{2 p(x)}}}\right)|\nabla u|^{p(x)-2} \nabla u\right)=\lambda f(x, u), \text { a.e. in } \Omega, \\ u=0, \text { on } \partial \Omega,\end{array}\right.$

where $\Omega \subset \mathrm{R}^{N}$ is a bounded domain with smooth boundary $\partial \Omega, \lambda>0$ is a parameter. The purpose of this paper is to obtain the existence results of nontrivial solutions for every parameter $\lambda$. Firstly, by using the mountain pass theorem a nontrivial solution is constructed for almost every parameter $\lambda>0$. Then we consider the continuation of the solutions. Our results are a generalization of that of Manuela Rodrigues.
\end{abstract}

\section{Introduction}

During the last fifteen years, variational problems and partial differential equations with various types of nonstandard growth conditions have become increasingly popular. This is partly due to their frequent appearance in applications such as the modeling of electrorheological fluids $[1,12]$ and image processing [2], but these problems are very interesting from a purely mathematical point of view as well.

In this paper, we consider the following nonlinear eigenvalue problems for $p(x)$-Laplacian-like operators originated from a capillary phenomena of the following form:

$$
(P)\left\{\begin{array}{l}
-\operatorname{div}\left(\left(1+\frac{|\nabla u|^{p(x)}}{\sqrt{1+|\nabla u|^{2 p(x)}}}\right)|\nabla u|^{p(x)-2} \nabla u\right)=\lambda f(x, u), \text { a.e. in } \Omega, \\
u=0, \text { on } \partial \Omega
\end{array}\right.
$$

Received July 31, 2012; Revised April 5, 2013.

2010 Mathematics Subject Classification. Primary 35D05; Secondary 35J70.

Key words and phrases. superlinear problem, $p(x)$-Laplacian, variational method, variable exponent Sobolev space.

Supported by the National Science Found of China (nos.11201095, 11126286, 11001063), the Fundamental Research Funds for the Central Universities, China Postdoctoral Science Foundation Funded Project (no. 20110491032), China Postdoctoral Science (Special) Foundation (no. 2012T50303). 
where $\Omega \subset \mathrm{R}^{N}$ is a bounded domain with smooth boundary $\partial \Omega, \lambda>0$ is a parameter.

M. Manuela Rodrigues [11] established the existence of nontrivial solution of problem $(P)$, by assuming the following conditions:

$\left(f_{1}\right) f: \Omega \times \mathbb{R} \rightarrow \mathbb{R}$ satisfies Caratheodory condition and

$$
|f(x, t)| \leq c_{1}+c_{2}|t|^{\beta(x)-1}, \quad \forall(x, t) \in \Omega \times \mathbb{R},
$$

where $\beta \in C_{+}(\bar{\Omega})$ and $1<\beta(x)<p^{*}(x)$ for $x \in \bar{\Omega}, p^{*}(x)=\frac{N p(x)}{N-p(x)}$ if $p(x)<N$, $p^{*}(x)=\infty$ if $p(x) \geq N$.

$\left(f_{2}\right) \exists M>0, \theta>p^{+}$such that

$$
0<\theta F(x, t) \leq t f(x, t), \quad \forall|t| \geq M, x \in \Omega,
$$

where $F(x, t)=\int_{0}^{t} f(x, s) d s$.

$\left(f_{3}\right) f(x, t)=o\left(|t|^{p^{+}-1}\right), t \rightarrow 0$ for $x \in \Omega$ uniformly and $\beta^{-}>p^{+}$.

It is well known, condition $\left(f_{2}\right)$ is quite important not only to ensure that the Euler-lagrange functional associated to problem $(P)$ has a mountain pass geometry, but also to guarantee that Palais-Smale sequence of the Euler-Lagrange functional is bounded. But this condition is very restrictive eliminating many nonlinearities. We recall that $\left(f_{2}\right)$ implies a weaker condition

$$
F(x, t) \geq c_{3}|t|^{\theta}-c_{4}, \quad c_{3}, c_{4}>0,(x, t) \in \Omega \times \mathbb{R} \text { and } \theta>p^{+} .
$$

The above condition implies another much weaker condition, which is a consequence of the superlinearity of $f$ at infinity:

$\left(f_{4}\right) \lim _{|t| \rightarrow \infty} \frac{F(x, t)}{|t|^{p^{+}}}=+\infty$, uniformly a.e. $x \in \Omega$.

Because the $p(x)$-Laplacian possesses more complicated nonlinearities than Laplacian and $p$-Laplacian, for example, it is inhomogeneous, thus our problem is much more difficult.

The main result of this paper is the following theorem.

Theorem 1.1 (Main Theorem). Under hypotheses $\left(f_{1}\right),\left(f_{3}\right),\left(f_{4}\right)$ and

$\left(f_{5}\right)$ there exists $t_{0}>0$, such that $\frac{f(x, t)}{t^{2 p^{+}-1}}$ is increasing in $t \geq t_{0}$ and decreasing in $t \leq-t_{0}, \forall x \in \Omega$.

Moreover, $f \in C(\bar{\Omega} \times \mathbb{R})$, then problem $(P)$ has a nontrivial weak solution, for all $\lambda>0$.

Example. Function $f(x, t)=\left(\beta(x) \ln \left(\frac{t}{3}\right)+3\right) t^{\beta(x)-1}\left(F(x, t)=t^{\beta(x)} \ln \left(\frac{t}{3}\right)\right)$ where $\beta \in C_{+}(\bar{\Omega})$ satisfies condition $\left(f_{5}\right)$, but it does not satisfy $\left(f_{2}\right)$ if $2 \beta^{-}>$ $p^{+}>\beta^{+}$.

Remark 1.2. In fact our result still holds if we consider a weaker condition than $\left(f_{5}\right)$, which is,

$\left(f_{5}\right)^{\prime}$ there is $C_{*}>0$ such that $f(x, t) t-\theta F(x, t) \leq f(x, s) s-\theta F(x, s)+C_{*}$ for all $0<t<s$ or $s<t<0$. 
This paper is organized in the following way: in Section 2, we recall some necessary preliminaries, which will be used in our investigation in Section 3; In Section 3, we prove the main result of the paper.

\section{Preliminaries}

In this part, we introduce some definitions and results which will be used in the next section.

Firstly, we introduce some theories of Lebesgue-Sobolev space with variable exponent. The detailed description can be found in $[3,4,6,8,7,10]$.

Write

$$
\begin{aligned}
& C_{+}(\bar{\Omega})=\{h \in C(\bar{\Omega}): h(x)>1 \text { for any } x \in \bar{\Omega}\}, \\
& h^{-}=\min _{x \in \bar{\Omega}} h(x), h^{+}=\max _{x \in \bar{\Omega}} p(x) \text { for any } h \in C_{+}(\bar{\Omega}) .
\end{aligned}
$$

Obviously, $1<h^{-} \leq h^{+}<+\infty$.

Denote by $\mathcal{U}(\Omega)$ the set of all measurable real functions defined on $\Omega$. Two functions in $\mathcal{U}(\Omega)$ are considered to be one element of $\mathcal{U}(\Omega)$, when they are equal almost everywhere.

For $p \in C_{+}(\bar{\Omega})$, define

$$
L^{p(x)}(\Omega)=\left\{u \in \mathcal{U}(\Omega): \int_{\Omega}|u(x)|^{p(x)} d x<+\infty\right\},
$$

with the norm $|u|_{L^{p(x)}(\Omega)}=|u|_{p(x)}=\inf \left\{\lambda>0: \int_{\Omega}\left|\frac{u(x)}{\lambda}\right|^{p(x)} d x \leq 1\right\}$, and

$$
W^{1, p(x)}(\Omega)=\left\{u \in L^{p(x)}(\Omega):|\nabla u| \in L^{p(x)}(\Omega)\right\}
$$

with the norm $\|u\|_{W^{1, p(x)}(\Omega)}=|u|_{p(x)}+|\nabla u|_{p(x)}$.

Denote $W_{0}^{1, p(x)}(\Omega)$ as the closure of $C_{0}^{\infty}(\Omega)$ in $W^{1, p(x)}(\Omega)$.

We remember that the variable exponent Lebesgue spaces are separable and reflexive Banach spaces. Denote by $L^{q(x)}(\Omega)$ the conjugate Lebesgue space of $L^{p(x)}(\Omega)$ with $\frac{1}{p(x)}+\frac{1}{q(x)}=1$, then the Hölder type inequality

$$
\int_{\Omega}|u v| d x \leq\left(\frac{1}{p^{-}}+\frac{1}{q^{-}}\right)|u|_{p(x)}|v|_{q(x)}, \quad u \in L^{p(x)}(\Omega), v \in L^{q(x)}(\Omega)
$$

holds. Furthermore, define mapping $\rho: L^{p(x)}(\Omega) \rightarrow \mathbb{R}$ by

$$
\rho(u)=\int_{\Omega}|u(x)|^{p(x)} d x,
$$

then the following relations hold

$$
\begin{aligned}
|u|_{p(x)}>1 \Rightarrow|u|_{p(x)}^{p^{-}} \leq \rho(u) \leq|u|_{p(x)}^{p^{+}} & \\
|u|_{p(x)}<1 & \Rightarrow|u|_{p(x)}^{p^{+}} \leq \rho(u) \leq|u|_{p(x)}^{p^{-}}
\end{aligned}
$$

Proposition $2.1([7])$. In $W_{0}^{1, p(x)}(\Omega)$ the Poincare's inequality holds, that is, there exists a positive constant $C_{0}$ such that

$$
|u|_{p(x)} \leq C_{0}|\nabla u|_{p(x)}, \quad \forall u \in W_{0}^{1, p(x)}(\Omega) .
$$


So $|\nabla u|_{p(x)}$ is an equivalent norm in $W_{0}^{1, p(x)}(\Omega)$. We will use the equivalent norm in the following discussion and write $\|u\|=|\nabla u|_{p(x)}$ for simplicity.

Proposition 2.2 ([5]). If $q \in C_{+}(\bar{\Omega})$ and $q(x)<p^{*}(x)$ for any $x \in \bar{\Omega}$, then the embedding from $W^{1, p(x)}(\Omega)$ to $L^{q(x)}(\Omega)$ is compact and continuous.

Consider the following function:

$$
J(u)=\int_{\Omega} \frac{1}{p(x)}\left(|\nabla u|^{p(x)}+\sqrt{1+|\nabla u|^{2 p(x)}}\right) d x, u \in W_{0}^{1, p(x)}(\Omega) .
$$

We denote $A=J^{\prime}: W_{0}^{1, p(x)}(\Omega) \rightarrow\left(W_{0}^{1, p(x)}(\Omega)\right)^{*}$, then

$$
\langle A(u), v\rangle=\int_{\Omega}\left(|\nabla u|^{p(x)-2}+\frac{|\nabla u|^{2 p(x)-2}}{\sqrt{1+|\nabla u|^{2 p(x)}}}\right)(\nabla u, \nabla v)_{\mathbb{R}^{N}} d x
$$

for all $u, v \in W_{0}^{1, p(x)}(\Omega)$.

Proposition $2.3([1])$. Set $X=W_{0}^{1, p(x)}(\Omega), A$ is as above, then

(1) $A: X \rightarrow X^{*}$ is a convex, bounded and strictly monotone operator;

(2) $A: X \rightarrow X^{*}$ is a mapping of type $(S)_{+}$, i.e., if $u_{n} \stackrel{w}{\rightarrow} u$ in $X$ and $\lim \sup \left\langle A\left(u_{n}\right), u_{n}-u\right\rangle \leq 0$, implies $u_{n} \rightarrow u$ in $X$;

(3) $A: X \rightarrow X^{*}$ is a homeomorphism.

\section{Existence theorems}

Now we introduce the energy functional $\varphi: W_{0}^{1, p(x)}(\Omega) \rightarrow \mathrm{R}$ associated with problem $(P)$, defined by

$$
\begin{aligned}
\varphi_{\lambda}(u)= & \int_{\Omega} \frac{1}{p(x)}\left(|\nabla u|^{p(x)}+\sqrt{1+|\nabla u|^{2 p(x)}}\right) d x \\
& -\lambda \int_{\Omega} F(x, u) d x, u \in W_{0}^{1, p(x)}(\Omega),
\end{aligned}
$$

which is due to [11].

From the hypotheses on $f$, it is standard to check that $\left.\varphi_{\lambda} \in C^{1}\left(W_{0}^{1, p(x)}(\Omega), \mathbb{R}\right)\right)$ and its Gateaux derivative is

$$
\begin{aligned}
\left\langle\varphi_{\lambda}^{\prime}(u), v\right\rangle= & \int_{\Omega}\left(|\nabla u|^{p(x)-2}+\frac{|\nabla u|^{2 p(x)-2}}{\sqrt{1+|\nabla u|^{2 p(x)}}}\right)(\nabla u, \nabla v)_{\mathbb{R}^{N}} d x \\
& -\lambda \int_{\Omega} f(x, u) v d x, u, v \in W_{0}^{1, p(x)}(\Omega) .
\end{aligned}
$$

Lemma 3.1. (a) Under the condition $\left(f_{4}\right)$, the functional $\varphi_{\lambda}$ is unbounded from below.

(b) Under the conditions $\left(f_{1}\right)$ and $\left(f_{3}\right), u=0$ is a strict local minimum for $\varphi_{\lambda}$ 
Proof. (a) From $\left(f_{4}\right)$, it is follows, for all $M>0$ there exists $C_{M}>0$, such that

$$
F(x, t) \geq M t^{p^{+}}-C_{M}, \forall x \in \Omega, t \geq 0 .
$$

Take $\phi \in C_{0}^{\infty}(\Omega) \backslash\{0\}$ with $\phi(x)>0$. For $t>1$, from (3.1) we have

$$
\begin{aligned}
\varphi_{\lambda}(t \phi)= & \int_{\Omega} \frac{1}{p(x)}\left(|\nabla t \phi|^{p(x)}+\sqrt{1+|\nabla t \phi|^{2 p(x)}}\right) d x-\lambda \int_{\Omega} F(x, t \phi) d x \\
\leq & t^{p^{+}} \int_{\Omega}\left(|\nabla \phi|^{p(x)}+\sqrt{1+|\nabla \phi|^{2 p(x)}}\right) d x-\lambda M t^{p^{+}} \int_{\Omega}|\phi|^{p^{+}} d x \\
& +\lambda C_{M}|\Omega| \\
\leq & t^{p^{+}} \int_{\Omega}\left(|\nabla \phi|^{p(x)}+\sqrt{1+|\nabla \phi|^{2 p(x)}}\right) d x-\lambda M t^{p^{+}} \int_{\Omega}|\phi|^{p^{+}} d x \\
& +\lambda C_{M}|\Omega| \\
= & t^{p^{+}}\left[\int_{\Omega}\left(|\nabla \phi|^{p(x)}+\sqrt{1+|\nabla \phi|^{2 p(x)}}\right) d x-\lambda M \int_{\Omega}|\phi|^{p^{+}} d x\right] \\
& +\lambda C_{M}|\Omega|,
\end{aligned}
$$

where $|\Omega|$ denotes the Lebesgue measure of $\Omega$. If $M$ is large, then

$$
\lim _{t \rightarrow \infty} \varphi_{\lambda}(t \phi)=-\infty
$$

This proves (a).

(b) From $\left(f_{3}\right)$, for any $\varepsilon>0$, there exists $\delta=\delta(\varepsilon)>0$, such that

$$
F(x, t) \leq \varepsilon|t|^{p^{+}}, \quad \forall t \in(-\delta, \delta), x \in \Omega .
$$

On the other hard, by $\left(f_{1}\right)$ and the mean value theorem, there exists $c_{5}>0$ such that,

$$
F(x, t) \leq c_{5}|t|^{\beta(x)} \quad \text { for a.e. } x \in \Omega \text { and }|t| \geq \delta .
$$

Therefore, it is follows that

$$
F(x, t) \leq \varepsilon|t|^{p^{+}}+c_{5}|t|^{\beta(x)} \quad \text { for a.e. } x \in \Omega \text { and all } t \in \mathrm{R} .
$$

For $u \in W_{0}^{1, p}(\Omega)$ and $\|u\|<1$, we have

$$
\begin{aligned}
\varphi_{\lambda}(u) & =\int_{\Omega} \frac{1}{p(x)}\left(|\nabla u|^{p(x)}+\sqrt{1+|\nabla u|^{2 p(x)}}\right) d x-\lambda \int_{\Omega} F(x, u) d x \\
& \geq \frac{2}{p^{+}} \int_{\Omega}|\nabla u|^{p(x)} d x-\lambda \varepsilon \int_{\Omega}|u|^{p^{+}} d x-\lambda c_{5} \int_{\Omega}|u|^{\beta(x)} d x \\
& \geq \frac{2}{p^{+}}\|u\|^{p^{+}}-\lambda \varepsilon C_{0}^{p^{+}}\|u\|^{p^{+}}-c_{5} C_{0}^{\beta^{-}}\|u\|^{\beta^{-}}
\end{aligned}
$$

here we use the continuity(in fact, compactness) embedding $W_{0}^{1, p(x)}(\Omega)$ into $L^{\beta(x)}(\Omega)$ (recall that $1<\beta(x)<p^{*}(x)$ ) and Poincare's inequality. Then

$$
\varphi_{\lambda}(x) \geq\left(\frac{2}{p^{+}}-\lambda \varepsilon C_{0}^{p^{+}}\right)\|u\|^{p^{+}}-\lambda c_{5} C_{0}^{\beta^{-}}\|u\|^{\beta^{-}} .
$$


For given $\lambda>0$, we choose $\varepsilon=\varepsilon(\lambda)>0$, such that $\varepsilon<\frac{1}{\lambda C_{0}^{p^{+}} p^{+}}$. Then from (3.2) and Poincare's inequality we have

$$
\varphi_{\lambda}(x) \geq \frac{1}{p^{+}}\|u\|^{p^{+}}-c_{6}\|u\|^{\beta^{-}}
$$

for some $c_{6}>0$ and all $u \in W_{0}^{1, p(x)}(\Omega)$.

Since $p^{+}<\beta^{-}$, if we choose $\rho=\rho(\lambda)>0$ small, from (3.3), we see that $\left\{\varphi_{\lambda}(u):\|u\|=\rho\right\} \geq d(\lambda)>0$. So far, we complete the proof.

Fix $0<\lambda_{0}<\mu_{0}$. Now we can see that geometry on $\varphi_{\lambda}$ works uniformly on $\left[\lambda_{0}, \mu_{0}\right]$. By choosing $\varepsilon>0$ such that $\frac{2}{p^{+}}-\mu_{0} \varepsilon C_{0}^{p^{+}} \geq \frac{1}{p^{+}}$, we obtain that

$$
\varphi_{\lambda}(u) \geq \frac{1}{p^{+}}\|u\|^{p^{+}}-c_{7}\|u\|^{\beta^{-}}, \forall u \in W_{0}^{1, p(x)}(\Omega), 0<\lambda \leq \mu_{0}, c_{7}>0 .
$$

That is, there exist $\rho>0$ and $r>0$, such that

$$
\varphi_{\lambda}(u) \geq r,\|u\|=\rho, \forall \lambda \leq \mu_{0} .
$$

By choosing $e \in W_{0}^{1, p(x)}(\Omega)$, such that $\varphi_{\lambda_{0}}(e)<0$, we infer that

$$
\frac{\varphi_{\lambda}(e)}{\lambda}<\frac{\varphi_{\lambda_{0}}(e)}{\lambda_{0}}<0, \lambda_{0} \leq \lambda \leq \mu_{0}
$$

Also we have

$$
\frac{\varphi_{\lambda}(u)}{\lambda} \leq \frac{\varphi_{\mu}(u)}{\mu}, \forall u \in W_{0}^{1, p(x)}(\Omega), \mu<\lambda .
$$

\section{Define}

$$
\mathcal{T}=:\left\{\gamma:[0,1] \rightarrow W_{0}^{1, p(x)}(\Omega) \mid \gamma \text { is continuous and } \gamma(0)=0 \text { and } \gamma(1)=e\right\}
$$

and for $\lambda_{0} \leq \lambda \leq \mu_{0}$, let $c_{\lambda}=: \inf _{\gamma \in \mathcal{T}} \max _{t \in[0,1]} \varphi_{\lambda}(\gamma(t))$.

We recall that the map $c:\left[\lambda_{0}, \mu_{0}\right] \rightarrow \mathrm{R}_{+}$, given by $c(\lambda)=c_{\lambda}$, is bounded from below by $c_{\mu_{0}}>0$.

In fact, (3.5) implies the monotonicity of $\frac{c_{\lambda}}{\lambda}$, while the estimate (3.4) implies $c_{\lambda} \geq r>0$.

Now, we are in the position to check the left semi-continuity of $\frac{c_{\lambda}}{\lambda}$. Fix $\mu \in\left[\lambda_{0}, \mu_{0}\right]$ and $\varepsilon>0$. Then fix $\gamma \in \mathcal{T}$ such that

$$
c(\mu) \leq \max _{t \in[0,1]} \varphi_{\mu}(\gamma(t)) \leq c(\mu)+\frac{\varepsilon \mu}{8} .
$$

Let $R_{0}=\max _{t \in[0,1]}\left|\int_{\Omega} F(x, \gamma(t)) d x\right|$. Then, for $\lambda>\frac{\mu}{2}$ such that $\frac{1}{\lambda}<\frac{1}{\mu}+\frac{\varepsilon}{2 c_{\mu}}$, we have

$$
\begin{aligned}
\varphi_{\lambda}(\gamma(t)) & =\left(\varphi_{\lambda}(\gamma(t))-\varphi_{\mu}(\gamma(t))\right)+\varphi_{\mu}(\gamma(t)) \\
& \leq \varphi_{\mu}(\gamma(t))+(\mu-\lambda) \int_{\Omega} F(x, \gamma(t)) d x \\
& \leq R_{0}|\lambda-\mu|+c_{\mu}+\frac{\varepsilon \mu}{8}, \forall t \in[0,1] .
\end{aligned}
$$


That is, $c_{\lambda} \leq c_{\mu}+\frac{\varepsilon \mu}{4}$, if $|\mu-\lambda|<\frac{\varepsilon \mu}{8 R_{0}}$. Hence, if $\mu>\lambda$, it follows that

$$
\frac{c_{\mu}}{\mu}-\varepsilon<\frac{c_{\mu}}{\mu} \leq \frac{c_{\lambda}}{\lambda} \leq \frac{c_{\mu}}{\lambda}+\frac{\varepsilon}{2} \leq \frac{c_{\mu}}{\mu}+\varepsilon
$$

Lemma $3.2([9])$. There exists $K>0$, such that

$$
\left\|\varphi_{\mu}^{\prime}(u)-\varphi_{\lambda}^{\prime}(u)\right\|_{\left(W_{0}^{1, p(x)}(\Omega)\right)^{*}} \leq K\left(1+\|u\|^{\beta^{+}-1}\right)|\mu-\lambda|, \forall \lambda, \mu>0 .
$$

Proof. For $\beta \in C_{+}(\bar{\Omega})$, define $\beta^{\prime}(x)$ such that $\frac{1}{\beta(x)}+\frac{1}{\beta^{\prime}(x)}=1, \forall x \in \bar{\Omega}$. By $\left(f_{1}\right)$, we have

$$
|f(x, t)|^{\beta^{\prime}(x)}=|f(x, t)|^{\frac{\beta(x)}{\beta(x)-1}} \leq d_{1}+d_{2}|t|^{\beta(x)}, \forall x \in \Omega, t \in \mathbb{R}
$$

for some constants $d_{1}, d_{2}>0$, and then

$$
\int_{\Omega}|f(x, t)|^{\beta^{\prime}(x)} d x \leq d_{1}|\Omega|+d_{2} \int_{\Omega}|u|^{\beta(x)} d x .
$$

Therefore, for any $u \in W_{0}^{1, p(x)}(\Omega)$, we have

$$
\int_{\Omega}|f(x, t)|^{\beta^{\prime}(x)} d x \leq d_{3}+d_{4}\|u\|^{\beta^{+}},
$$

where $d_{3}$ and $d_{4}$ are positive constants.

Thus, for all $v \in W_{0}^{1, p(x)}(\Omega)$ with $\|v\| \leq 1$, we have

$$
\left|\left\langle\varphi_{\mu}^{\prime}(u), v\right\rangle-\left\langle\varphi_{\lambda}^{\prime}(u), v\right\rangle\right| \leq(\lambda-\mu) \int_{\Omega} f(x, u) v d x .
$$

Thus,

$$
\begin{aligned}
\left|\left\langle\varphi_{\mu}^{\prime}(u), v\right\rangle-\left\langle\varphi_{\lambda}^{\prime}(u), v\right\rangle\right| & \leq|\lambda-\mu| \int_{\Omega}|f(x, u) \| v| d x \\
& \leq 2|\lambda-\mu||f(x, u)|_{\beta^{\prime}(x)}|v|_{\beta(x)} \\
& \leq 2 C_{0}|\lambda-\mu|\left(d_{3}+d_{4}\|u\|^{\beta^{+}}\right)^{\frac{\beta^{+}-1}{\beta^{+}}}\|v\| .
\end{aligned}
$$

So there exists constant $K>0$ such that

$$
\left\|\varphi_{\mu}^{\prime}(u)-\varphi_{\lambda}^{\prime}(u)\right\|_{\left(W_{0}^{1, p(x)}(\Omega)\right)^{*}} \leq K\left(1+\|u\|^{\beta^{+}-1}\right)|\mu-\lambda|, \forall \lambda, \mu>0 .
$$

Remark 3.3. We recall that the map $b:\left[\lambda_{0}, \mu_{0}\right] \rightarrow \mathrm{R}_{+}$, given by $b(\lambda)=\frac{c_{\lambda}}{\lambda}$, is monotone decreasing. Thus, $b(\lambda)$ and $c(\lambda)$ are differentiable at almost all value $\lambda \in\left(\lambda_{0}, \mu_{0}\right)$.

The proof of the next lemma is done by adapting some arguments employed in the proof of Lemma 3.3 in [13] and Lemma 2.5 in [14].

Lemma 3.4. Suppose the map $c:\left[\lambda_{0}, \mu_{0}\right] \rightarrow \mathbb{R}_{+}$, given by $c(\lambda)=c_{\lambda}$, is differentiable in $\mu$, then there exists a sequence $\left\{u_{n}\right\} \subset W_{0}^{1, p(x)}(\Omega)$ such that

$$
\varphi_{\mu}\left(u_{n}\right) \rightarrow c_{\mu}, \varphi_{\mu}^{\prime}\left(u_{n}\right) \rightarrow 0, \text { and }\left\|u_{n}\right\|^{p^{0}} \leq \bar{C} \text { as } n \rightarrow \infty,
$$


where $p^{0}=p^{+}$if $\left\|u_{n}\right\| \leq 1, p^{0}=p^{-}$if $\left\|u_{n}\right\|>1$, and $\bar{C}=p^{+} c_{\mu}+p^{+} \mu(2-$ $\left.c^{\prime}(\mu)\right)+1$.

Proof. Assume, by contradiction, that the lemma was false. Then $\left\|\varphi_{\mu}^{\prime}\left(u_{n}\right)\right\|_{*} \geq$ $2 \delta$ for all $u \in N_{\delta}^{\mu}=\left\{u \in W_{0}^{1, p(x)}(\Omega):\|u\|^{p^{0}} \leq \bar{C},\left|\varphi_{\mu}(u)-c_{\mu}\right| \leq \delta\right\}$.

Let $c_{8}$ be such that

$$
\begin{aligned}
\left|\int_{\Omega} F(x, u(x)) d x\right| & =\left|\varphi_{\mu}(u)-\int_{\Omega} \frac{1}{p(x)}\left(|\nabla u|^{p(x)}+\sqrt{1+|\nabla u|^{2 p(x)}}\right) d x\right| \\
& \leq\left|\varphi_{\mu}(u)\right|+\left|\int_{\Omega} \frac{1}{p(x)}\left(|\nabla u|^{p(x)}+\sqrt{1+|\nabla u|^{2 p(x)}}\right) d x\right| \\
& \leq \frac{1}{\mu}\left[c_{\mu}+\delta+\frac{1}{p^{-}} \int_{\Omega}\left(2|\nabla u|^{p(x)}+1\right) d x\right] \\
& \leq \frac{1}{\mu}\left[c_{\mu}+\delta+\frac{1}{p^{-}}\left(|\Omega|+2 \|\left. u\right|^{p^{0}}\right)\right] \\
& \leq c_{8}, \forall u \in N_{\delta}^{\mu} .
\end{aligned}
$$

Set $V: N_{\delta}^{\mu} \rightarrow W_{0}^{1, p}(\Omega)$ to be a locally Lipschtz pseudo-gradient vector field, $\|V\| \leq 1$ and

$$
\left\langle\varphi_{\mu}^{\prime}(u), V(u)\right\rangle \leq-\delta, \forall u \in N_{\delta}^{\mu} \quad(\text { see }[10]) .
$$

Now, fix $\left\{\lambda_{n}\right\}$ a sequence in $\left(\lambda_{0}, \mu_{0}\right)$ such that $\mu<\lambda_{n+1}<\lambda_{n}$, and $\lambda_{n}$ converges to $\mu,\left|\lambda_{n}-\mu\right| \leq \min \left\{\frac{\delta}{2 \mathrm{c}}, \frac{\delta}{2}\right\}$, and $\left|c_{\mu}-c_{\lambda_{n}}\right| \leq \frac{\delta}{4}$.

For each $n$, let $\gamma_{n} \in \mathcal{T}$ be such that

$$
\max _{t \in[0,1]} \varphi_{\mu}\left(\gamma_{n}(t)\right) \leq c_{\mu}+\left(\lambda_{n}-\mu\right) .
$$

Consider the open set

$$
A_{n}=\left\{t \in[0,1]: \varphi_{\lambda_{n}}\left(\gamma_{n}(t)\right)>c_{\lambda_{n}}-\left(\lambda_{n}-\mu\right)\right\}
$$

By the definition of $c_{\lambda_{n}}$, we know that $A_{n}$ is nonempty.

If $v \in \gamma_{n}\left(A_{n}\right)$, then from (3.7), we have,

$$
\int_{\Omega} F(x, v) d x=\frac{\varphi_{\mu}(v)-\varphi_{\lambda_{n}}(v)}{\lambda_{n}-\mu} \leq \frac{c_{\mu}-c_{\lambda_{n}}}{\lambda_{n}-\mu}+2=-c^{\prime}(\mu)+2+o_{n}(1),
$$

where we have used $c_{\mu}-c_{\lambda_{n}}=\left(c^{\prime}(\mu)+o_{n}(1)\right)\left(\mu-\lambda_{n}\right)$.

Since

$$
\int_{\Omega} \frac{1}{p(x)}\left(|\nabla u|^{p(x)}+\sqrt{1+|\nabla u|^{2 p(x)}}\right) d x=\varphi_{\mu}(v)+\mu \int_{\Omega} F(x, v) d x
$$

and

$$
\int_{\Omega} \frac{1}{p(x)}\left(|\nabla u|^{p(x)}+\sqrt{1+|\nabla u|^{2 p(x)}}\right) d x \geq \frac{1}{p^{+}} \int_{\Omega}|\nabla u|^{p(x)} d x \geq \frac{1}{p^{+}}\|v\|^{p^{0}},
$$


we obtain for $v \in \gamma_{n}\left(A_{n}\right)$,

$$
\begin{aligned}
\|v\|^{p^{0}} & \leq c_{\mu}+\left(\lambda_{n}-\mu\right)+\mu\left(-c^{\prime}(\mu)+2+o_{n}(1)\right) \\
& =p^{+} c_{\mu}+p^{+} \mu\left(2-c^{\prime}(\mu)\right)+p^{+}\left(\lambda_{n}-\mu\right)+\mu o_{n}(1) \\
& \leq \bar{C}
\end{aligned}
$$

for $n$ large.

It is easy to see that inequality (3.7) is satisfied for $v \in \gamma_{n}\left(A_{n}\right)$. Thus $\gamma_{n}\left(A_{n}\right) \subseteq N_{\delta}^{\mu}$, since,

$$
\begin{gathered}
c_{\lambda_{n}}-\left(\lambda_{n}-\mu\right) \leq \varphi_{\lambda_{n}}(v), \varphi_{\mu}(v) \leq c_{\mu}+\left(\lambda_{n}-\mu\right) . \\
\left|\varphi_{\lambda_{n}}(v)-\varphi_{\mu}(v)\right|=\left(\lambda_{n}-\mu\right)\left|\int_{\Omega} F(x, v) d x\right| \leq c_{8}\left|\lambda_{n}-\mu\right|,
\end{gathered}
$$

for $n$ large

$$
c_{\mu}-\delta<\varphi_{\mu}(v)<c_{\mu}+\delta, \forall v \in \gamma_{n}\left(A_{n}\right)
$$

So,

$$
\left\langle\varphi_{\lambda_{n}}^{\prime}(u), V(u)\right\rangle \leq-\frac{\delta}{2} \text { for all } u \in N_{\delta}^{\mu} .
$$

Now consider a Lipschitz continuous cut-off function $\eta$ such that $0 \leq \eta \leq$ $1 ; \eta(x)=0, x \notin N_{\delta}^{\mu} ; \eta(x)=1, x \in N_{\frac{\delta}{2}}^{\mu}$.

Let $\phi$ be the flow generated by $\eta V$, that is

$$
\left\{\begin{array}{l}
\frac{\partial \phi(u, r)}{\partial r}=\eta(\phi(u, r)) V(\phi(u, r)), \quad \text { on } \mathrm{R}_{+} \\
\phi(u, 0)=u
\end{array}\right.
$$

From the uniqueness result of ODE we have:

If $u \notin N_{\delta}^{\mu}$, then $\phi(u, r)=u, \forall r \geq 0$;

If $u \in N_{\delta}^{\mu}$, then $\phi(u, r) \in N_{\delta}^{\mu}, \forall r \geq 0$.

$\Rightarrow$ (i) If $u \in W_{0}^{1, p}(\Omega)$, then $\left\langle\varphi_{\lambda_{n}}^{\prime}(\phi(u, r)), \frac{\partial \phi(u, r)}{\partial r}\right\rangle \leq 0$,

$\Rightarrow$ (ii) If $\phi(x, r) \in N_{\frac{\delta}{2}}^{\mu}, \forall r \in\left[0, r_{0}\right]$, then

$$
\varphi_{\lambda_{n}}(\phi(u, r)) \leq \varphi_{\lambda_{n}}(u)-\frac{\delta}{2} r_{0}
$$

Since $e \notin N_{\delta}^{\mu}$, we have $\phi(e, r)=e$ and $\phi(0, r)=0$, for all $r \geq 0$, and then $\phi(\gamma, r) \in \mathcal{T}$, for all real $r$ and $\gamma \in \mathcal{T}$.

This implies that $h_{n}(t)=\phi\left(\gamma_{n}(t), 1\right)$ is a continuous path in $\mathcal{T}$ such that $\varphi_{\lambda_{n}}\left(h_{n}(t)\right) \leq \varphi_{\lambda_{n}}\left(\gamma_{n}(t)\right)$, and then for its maximum point $s_{n} \in[0,1]$, we should have $s_{n} \in A_{n}$, and

$$
c_{\mu}-o_{n}(1)=c_{\lambda_{n}} \leq \max _{t \in[0,1]} \varphi_{\lambda_{n}}\left(h_{n}(t)\right)=\varphi_{\lambda_{n}}\left(h_{n}\left(s_{n}\right)\right) \leq \varphi_{\lambda}\left(\gamma_{n}\left(s_{n}\right)\right)-\frac{\delta}{2} .
$$

On the other hand, from (3.7) and (3.8), we have

$$
\varphi_{\lambda}\left(\gamma_{n}\left(s_{n}\right)\right) \leq \varphi_{\mu}\left(\gamma_{n}\left(s_{n}\right)\right)+c_{3}\left|\lambda_{n}-\mu\right| \leq c_{\mu}+\left(1+c_{3}\right)\left|\lambda_{n}-\mu\right|
$$


which is a contradiction.

The next theorem follows directly from Lemma 3.4.

Theorem 3.5. For almost all $\lambda>0, c_{\lambda}$ is a critical value for $\varphi_{\lambda}$.

Proof. From Lemma 3.4, $\left\{u_{n}\right\}_{n \geq 1}$ is bounded and so we may assume that $u_{n} \rightarrow u$ (weak convergence) in $W_{0}^{1, p(x)}(\Omega)$ and $u_{n} \rightarrow u$ in $L^{\beta(x)}(\Omega)$.

As above from the choice of $\left\{u_{n}\right\}_{n \geq 1} \subseteq W_{0}^{1, p(x)}(\Omega)$, we have $\int_{\Omega} f\left(x, u_{n}\right)\left(u_{n}-\right.$ $u) d x \rightarrow 0$, that is

$$
\left\langle A\left(u_{n}\right), u_{n}-u\right\rangle \rightarrow 0 \Rightarrow u_{n} \rightarrow u \text { in } W_{0}^{1, p(x)}(\Omega) .
$$

So, $\varphi_{\mu}(u)=c_{\mu}, \varphi_{\mu}^{\prime}(u)=0$. That is $u$ is a critical point of $\varphi_{\mu}$.

Theorem 3.6. For almost all $\lambda>0$, problem $(P)$ has a nontrivial weak solution.

Proof. As $c_{\lambda}$ is left continuous, from Lemma 3.4, for each $\mu>0$, we can fix sequences $\left\{u_{n}\right\} \subseteq W_{0}^{1, p(x)}(\Omega)$, and $\left\{\lambda_{n}\right\} \subseteq \mathrm{R}$, such that

$$
\begin{aligned}
& \lambda_{n} \rightarrow \mu, c_{\lambda_{n}} \rightarrow c_{\mu} \text { as } n \rightarrow \infty . \\
& \varphi_{\lambda_{n}}\left(u_{n}\right)=c_{\lambda_{n}}, \varphi_{\lambda_{n}}^{\prime}\left(u_{n}\right)=0 .
\end{aligned}
$$

We claim that $\left\{u_{n}\right\}$ is bounded. Suppose that this is not true. Then we can assume that $\left\|u_{n}\right\| \rightarrow+\infty$, as $n \rightarrow+\infty$. Set $w_{n}=\frac{u_{n}}{\left\|u_{n}\right\|}, n \geq 1$.

We may assume that

$$
\begin{aligned}
& w_{n} \rightarrow w \text { weakly in } W_{0}^{1, p(x)}(\Omega) ; \\
& w_{n} \rightarrow w \text { in } L^{p(x)}(\Omega) ; \\
& w_{n} \rightarrow w \text { in } L^{\beta(x)}(\Omega) ; \quad\left(\text { by } 1<\beta(x)<p^{*}(x)\right) \\
& w_{n}(x) \rightarrow w(x) \text { a.e. on } \Omega ;
\end{aligned}
$$

and $\left|w_{n}(x)\right| \leq h(x)$ a.e. on $\Omega$, for $n \geq 1$ and $h \in L^{p(x)}(\Omega)$.

Let $\Omega_{0}=\{x \in \Omega: w(x) \neq 0\}$. If $x \in \Omega_{0}$, then

$$
\lim _{n \rightarrow \infty} \frac{F\left(x, u_{n}(x)\right)}{\left|u_{n}(x)\right|^{p^{+}}} \frac{\left|u_{n}(x)\right|^{p^{+}}}{\left\|u_{n}\right\|^{p^{+}}}=\lim _{n \rightarrow \infty} \frac{F\left(x, u_{n}(x)\right)}{\left|u_{n}(x)\right|^{p^{+}}}\left|w_{n}(x)\right|^{p^{+}}=+\infty\left(\text { by }\left(f_{4}\right)\right) .
$$

Applying the Fatou's lemma, we have

$$
\lim _{n \rightarrow \infty} \int_{\Omega} \frac{F\left(x, u_{n}(x)\right)}{\left|u_{n}(x)\right|^{p^{+}}}\left|w_{n}(x)\right|^{p^{+}} \leq \frac{1}{\mu p^{-}} .
$$

We conclude that $\Omega_{0}$ has zero measure and $w=0$ a.e. in $\Omega$.

Let $\varphi_{\lambda_{n}}\left(t_{n} u_{n}\right)=\max _{t \in[0,1]} \varphi_{\lambda_{n}}\left(t u_{n}\right)$, we have

$$
\varphi_{\lambda_{n}}\left(t x_{n}\right) \leq \varphi_{\lambda_{n}}\left(t_{n} x_{n}\right)
$$


and $\varphi_{\lambda_{n}}^{\prime}\left(t_{n} u_{n}\right)=0$, hence $\left\langle\varphi_{\lambda_{n}}^{\prime}\left(t_{n} u_{n}\right), t_{n} u_{n}\right\rangle=0$, that is

$$
\int_{\Omega}\left[\left|t_{n} \nabla u_{n}\right|^{p(x)}+\frac{\left|t_{n} \nabla u_{n}\right|^{2 p(x)}}{\sqrt{1+\left|t_{n} \nabla u_{n}\right|^{2 p(x)}}}\right] d x=\lambda_{n} \int_{\Omega} t_{n} u_{n} f\left(x, t_{n} u_{n}\right) d x .
$$

Next, we show that

$$
\begin{aligned}
& \frac{1}{p(x)} \sqrt{1+\left|t_{n} \nabla u_{n}\right|^{2 p(x)}}-\frac{1}{2 p^{+}} \frac{\left|t_{n} \nabla u_{n}\right|^{2 p(x)}}{\sqrt{1+\left|t_{n} \nabla u_{n}\right|^{2 p(x)}}} \\
\leq & \frac{1}{p(x)} \sqrt{1+\left|\nabla u_{n}\right|^{2 p(x)}}-\frac{1}{2 p^{+}} \frac{\left|\nabla u_{n}\right|^{2 p(x)}}{\sqrt{1+\left|\nabla u_{n}\right|^{2 p(x)}}} .
\end{aligned}
$$

In order to prove this, we define the following functional $f:[0,1] \rightarrow \infty$ :

$$
f(t)=p_{1} \sqrt{1+a t^{p}}-p_{2} \frac{a t^{p}}{\sqrt{1+a t^{p}}},
$$

where $p, p_{1}, p_{2}, a$ are positive constants with $p_{1} \geq 2 p_{2}$ and $p>1$. Obviously, $f^{\prime}(t) \geq 0, \forall t \in[0,1]$. Thus we deduce that $f\left(t_{n}\right) \leq f(1)$, that is

$$
p_{1} \sqrt{1+a t_{n}^{p}}-p_{2} \frac{a t_{n}^{p}}{\sqrt{1+a t_{n}^{p}}} \leq p_{1} \sqrt{1+a}-p_{2} \frac{a}{\sqrt{1+a}} .
$$

Finally, we notice that by taking in (3.10) $p_{1}=\frac{1}{p(x)}, p_{2}=\frac{1}{2 p^{+}}$and $a=$ $\left|\nabla u_{n}\right|^{2 p(x)}$, we deduce that (3.9) holds true.

Therefore, from $\left(f_{5}\right)$ and $(3.9)$, we have

$$
\begin{aligned}
\varphi_{\lambda_{n}}\left(t u_{n}\right) \leq & \varphi_{\lambda_{n}}\left(t_{n} u_{n}\right)-\frac{1}{2 p^{+}}\left\langle\varphi_{\lambda_{n}}^{\prime}\left(t_{n} u_{n}\right), t_{n} u_{n}\right\rangle \\
= & \int_{\Omega}\left[\frac{1}{p(x)}-\frac{1}{2 p^{+}}\right]\left|t_{n} \nabla u_{n}\right|^{p(x)} d x+\int_{\Omega} \frac{1}{p(x)} \sqrt{1+\left|t_{n} \nabla u_{n}\right|^{2 p(x)}} d x \\
& +\lambda_{n} \int_{\Omega}\left[\frac{1}{2 p^{+}} t_{n} u_{n} f\left(x, t_{n} u_{n}\right)-F\left(x, t_{n} u_{n}\right)\right] d x \\
& -\frac{1}{2 p^{+}} \int_{\Omega} \frac{\left|t_{n} \nabla u_{n}\right|^{2 p(x)}}{\sqrt{1+\left|t_{n} \nabla u_{n}\right|^{2 p(x)}} d x} \\
\leq & \int_{\Omega}\left[\frac{1}{p(x)}-\frac{1}{2 p^{+}}\right]\left|\nabla u_{n}\right|^{p(x)} d x+\int_{\Omega} \frac{1}{p(x)} \sqrt{1+\left|\nabla u_{n}\right|^{2 p(x)}} d x \\
& +\lambda_{n} \int_{\Omega}\left[\frac{1}{2 p^{+}} t_{n} u_{n} f\left(x, t_{n} u_{n}\right)-F\left(x, t_{n} u_{n}\right)\right] d x \\
& -\frac{1}{2 p^{+}} \int_{\Omega} \frac{\left|\nabla u_{n}\right|^{2 p(x)}}{\sqrt{1+\left|\nabla u_{n}\right|^{2 p(x)}}} d x \\
\leq & \int_{\Omega}\left[\frac{1}{p(x)}-\frac{1}{2 p^{+}}\right]\left|\nabla u_{n}\right|^{p(x)} d x+\int_{\Omega} \frac{1}{p(x)} \sqrt{1+\left|\nabla u_{n}\right|^{2 p(x)}} d x \\
& +\lambda_{n} \int_{\Omega}\left[\frac{1}{2 p^{+}} u_{n} f\left(x, u_{n}\right)-F\left(x, u_{n}\right)+\frac{C_{*}}{2 p^{+}}\right] d x
\end{aligned}
$$




$$
\begin{aligned}
& -\frac{1}{2 p^{+}} \int_{\Omega} \frac{\left|\nabla u_{n}\right|^{2 p(x)}}{\sqrt{1+\left|\nabla u_{n}\right|^{2 p(x)}}} d x \\
= & c_{\lambda_{n}}+\frac{\lambda_{n} C_{*}}{2 p^{+}}|\Omega|
\end{aligned}
$$

for all $t \in[0,1]$.

On the other hard, for all $R>1$, set $R_{1}=(\theta R)^{\frac{1}{p^{-}}}$,

$$
\begin{aligned}
\varphi_{\lambda_{n}}\left(R_{1} w_{n}\right)= & \int_{\Omega} \frac{1}{p(x)}\left(\left|R_{1} \nabla w_{n}\right|^{p(x)}+\sqrt{1+\left|R_{1} \nabla w_{n}\right|^{2 p(x)}}\right) d x \\
& -\lambda_{n} \int_{\Omega} F\left(x, R_{1} w_{n}\right) d x \\
\geq & \frac{2}{\theta} R_{1}^{p^{-}}\left\|w_{n}\right\|^{p^{0}}-\lambda_{n} \int_{\Omega} F\left(x, R_{1} w_{n}\right) d x \\
= & 2 R-\lambda_{n} \int_{\Omega} F\left(x, R_{1} w_{n}\right) d x \\
\geq & R,
\end{aligned}
$$

which contradicts $\varphi_{\lambda_{n}}\left(R_{1} w_{n}\right) \leq c_{\lambda_{n}}+\frac{\lambda_{n} C_{*}}{\theta}|\Omega|$, for $n$ large.

Now we have a bounded sequence $\left\{x_{n}\right\}$ such that

$$
\varphi_{\mu}\left(x_{n}\right) \rightarrow c_{\mu} \quad \text { and } \quad \varphi_{\mu}^{\prime}\left(x_{n}\right)=0, \quad n \rightarrow \infty .
$$

The proof is done.

\section{References}

[1] E. Acerbi and G. Mingione, Regularity results for stationary electro-rheological fluids, Arch. Ration. Mech. Anal. 164 (2002), no. 3, 213-259.

[2] Y. Chen, S. Levine, and M. Rao, Variable exponent, linear growth functionals in image restoration, SIAM J. Appl. Math. 66 (2006), no. 4, 1383-1406.

[3] L. Diening, Riesz potential and Sobolev embedding on generalized Lebesque and Sobolev space $L^{p(\cdot)}$ and $W^{k, p(\cdot)}$, Math. Nachr. $268(2004), 31-43$.

[4] D. E. Edmunds and J. Rákosnic, Sobolev embbeding with variable exponent II, Math. Nachr. 246/247 (2002), 53-67.

[5] X. L. Fan and Q. H. Zhang, Existence of solutions for $p(x)$-Laplacian Dirichlet problem, Nonlinear Anal. 52 (2003), no. 8, 1843-1852.

[6] X. L. Fan and D. Zhao, On the generalized Orlicz-Sobolev spaces $W^{k, p(x)}(\Omega)$, J. Gansu Educ. College 12 (1998), no. 1, 1-6.

[7] $2,424-446$.

[8] X. L. Fan, Y. Z. Zhao, and D. Zhao, Compact imbedding theorems with symmetry of Strauss-Lions type for the space $W^{1, p(x)}(\Omega)$, J. Math. Anal. Appl. 255 (2001), no. 1, 333-348.

[9] C. Ji, On the superlinear problem involving the $p(x)$-Laplacian, Electron. J. Qual. Theory Differ. 40 (2011), 1-9.

[10] O. Kovacik and J. Rakosuik, On spaces $L^{p(x)}(\Omega)$ and $W^{k, p(x)}(\Omega)$, Czechoslovak Math. J. 41 (1991), no. 4, 592-618.

[11] M. M. Rodrigues, Multiplicity of solutions on a nonlinear eigenvalue problem for $p(x)$ Laplacian-like operators, Mediterr. J. Math. 9 (2012), no. 1, 211-222. 
[12] M. Ružička, Electrorheological Fluids: Modeling and Mathematical Theory, SpringerVerlag, Berlin, 2000.

[13] M. Struwe and G. Tarantello, On multivortex solutions in Chern-Simons gauge theory, Boll. Unione Mat. Ital. Sez. B Artic. Ric. Mat. (8) 1 (1998), no. 1, 109-121.

[14] G. Wang and J. Wei, Steady state solutions of a reaction-diffusion system modeling chemotaxis, Math. Nachr. 233/234 (2002), 221-236.

Department of Applied Mathematics

HARBIN ENGINEERING UNIVERSITY

Harbin, 150001, P. R. China

E-mail address: gebin04523080261@163.com 